\title{
Dosis Y Épocas De Aplicación De Nicosulfuron En El Cultivo De Maíz En La Localidad De Babahoyo, Ecuador
}

\author{
Luis Alcívar-Torres, Profesor Investigador \\ Dalton Cadena-Piedrahita, Profesor Investigador \\ Juan Salinas-Ochoa, Profesor \\ Miguel Goyes-Cabezas, Profesor \\ Danilo Santana-Aragone, Analista de Laboratorios \\ Universidad Técnica de Babahoyo, Ecuador
}

doi: 10.19044/esj.2016.v12n33p290 URL:http://dx.doi.org/10.19044/esj.2016.v12n33p290

\begin{abstract}
This study evaluated the effect of three doses of nicosulfuron postemergent herbicide(40, 50 y $60 \mathrm{gr} / \mathrm{ha})$, applied in three moments of the vegetative stage of hybrid corn "Trueno NB - 7443" (12, 18 and 24 days after sow), using two levels of fertilization (76-15 - 90 and $152-30-180$ of $\mathrm{N}-\mathrm{P}_{2} \mathrm{O}_{5}$ y $\mathrm{K}_{2} \mathrm{O}$ Kg., respectively) and one population (71.428 plants/ha). The experiment is performed on a total area of $1857.6 \mathrm{~m}^{2}$ and each experimental unit had an area of $16.8 \mathrm{~m}^{2}$; the experimental design was randomized complete block factorial arrangement of $2 \times 9$ with a total of 18 treatments and 4 repetitions. The toxicity index and other agronomic indicators of production depending on the dose and time of application of herbicide are determined. The results showed phytotoxicity on selectivity only 3 days evaluation after herbicide application in different doses. Treatment based on $40 \mathrm{~g} /$ ha of herbicide applied at $12 \mathrm{dds}$, in the presence of 76 levels and $152-15-90-30-180 \mathrm{~kg} /$ ha NPK, he had the lowest toxicity index and concluded that the levels of fertilization did not influence the susceptibility of the hybrid corn used.
\end{abstract}

Keywords: Weed, phytotoxicity, herbicide, hybrid, corn

\section{Resumen}

El objetivo este trabajo fue evaluar el efecto de tres dosis del herbicida post-emergente nicosulfuron (40, 50 y $60 \mathrm{gr} / \mathrm{ha}$ ), aplicados en tres momentos de la etapa vegetativa del híbrido de maíz "trueno NB - 7443" (12, 18 y 24 días después de la siembra), utilizando dos niveles de 
fertilización (76-15-90 y 152-30-180 $\mathrm{kg}$ de $\mathrm{N} \quad-\mathrm{P}_{2} \mathrm{O}_{5} \quad$ y $\quad \mathrm{K}_{2} \mathrm{O}$, respectivamente) y una sola población (71.428 plantas/ha). El experimento se efectuó sobre un área total de $1857,6 \mathrm{~m}^{2}$ y cada unidad experimental tuvo un área de $16,8 \mathrm{~m}^{2}$; se utilizó el diseño experimental de bloques completos al azar con arreglo factorial de 2 × 9 con un total de 18 tratamientos y 4 réplicas. Se determinó el índice de toxicidad y otros indicadores agronómicos de producción en función de las dosis y momento de la aplicación del herbicida. Los resultados sobre selectividad presentaron fitotoxicidad únicamente a los 3 días de evaluación, luego de la aplicación del herbicida en las diferentes dosis. El tratamiento basado en $40 \mathrm{~g} / \mathrm{ha}$ del herbicida aplicado a los 12 dds, en presencia de los niveles 76 -15- 90 y 152 -30 -180 kg/ha NPK, presentó el menor índice de toxicidad. Se concluyó que los niveles de fertilización no influyeron en la susceptibilidad del híbrido de maíz empleado.

Palabras-clave: Maleza, fitotoxicidad, herbicida, híbrido, maíz

\section{Introducción}

Globalmente el maíz (Zea mays L.) se siembra anualmente en una extensión de 40 millones de hectáreas, alcanzando una producción de más de 600 millones de toneladas de grano. Más del 50\% de la producción se utiliza en la alimentación de aves y cerdos, convirtiéndose, junto al arroz y soya en uno de los cultivos de ciclo corto mayormente sembrado. En Ecuador, se siembran aproximadamente 250 mil hectáreas, entre las cuales el $50 \%$ se ubica en la provincia de Los Ríos, el $40 \%$ en Manabí y el resto en Guayas; el $90 \%$ de la siembra de maíz se lo realiza en época lluviosa, con un rendimiento promedio de 2,23 t/ ha.

Tres cuartas partes de la producción total proviene de unidades familiares campesinas; la mayoría de ellas con economía de subsistencia, como también por constituir la principal materia prima para la elaboración de alimentos concentrados (balanceados) destinados a la industria animal.

Entre los factores limitantes de la productividad en el cultivo de maíz se encuentran las malezas, cuyo control efectivo permite obtener alta producción; sin embargo este control es el mal efectuado debido al desconocimiento del manejo de labores culturales, en particular el uso de los herbicidas. En los últimos años han aparecido en el mercado nacional nuevos herbicidas post emergentes como el Accent ${ }^{* 13}$ cuyo ingrediente de acción es el nicosulfuron; por lo cual se justificó la investigación basada en evaluar los

\footnotetext{
* La mención de la marca comercial del producto, obedece únicamente a propósitos de identificación, no existiendo ningún compromiso promocional con relación al mismo por parte de los autores del artículo.
} 
efectos de la aplicación del herbicida “Accent” en diferentes etapas vegetativas del híbrido de maíz duro 'Trueno NB - 7443'.

\section{Materiales y métodos}

La investigación se realizó en los terrenos de la Facultad de Ciencias Agropecuarias de la Universidad Técnica de Babahoyo entre el año 2010 y 2011 e incluyó el uso de los métodos deductivo-inductivo y el experimental. Se estudiaron tres factores: Dosis, épocas de aplicación del herbicida nicosulfuron y niveles de fertilización química.

El experimento estuvo conformado por 18 tratamientos y cuatro réplicas, con un total de 72 unidades experimentales, cuyas dimensiones fueron de 2,8m de ancho, el distanciamiento de siembra fue de0,70 m y 0,20 m (Coello y D. 2014). Se utilizó un diseño de Bloques Completos al Azar con arreglo factorial de 2 x 9. Todas las variables evaluadas fueron sometidas al análisis de varianza, para la comparación de las medias de los niveles de fertilización se empleó la prueba de Diferencia Mínima Significativa (DMS); y la prueba de Tukey al 95\% de confianza para comparar las medias de las dosis y épocas de aplicación del herbicida Accent y la interacción entre los dos factores estudiados.

Las dosis del herbicida Accent fueron: 40, 50 y $60 \mathrm{~g} / \mathrm{ha}$, las cuales están dentro de lo de lo recomendado por Hernández, Arreaza, y Lazo (2002) fueron aplicadas a los 12, 18 y 24 días después de la siembra, considerados los momentos del ciclo vegetativo en que el Nicosulfuron actúa eficientemente según el genotipo (Forti y P. 1995). Los niveles de fertilización química fueron: a) 76-15-90 y b) 152-30-180 kg/ ha de nitrógeno, fósforo y potasio, respectivamente; siendo la primera una experimentación insuficiente para satisfacer las necesidades nutricionales del cultivo, y la segunda, la recomendación para híbridos de este tipo como lo manifiesta Fallas et al. (2010) en función del análisis de suelo realizado.

La selectividad del herbicida, se realizó visualmente a los 3, 7, 14 y 21 días después de la aplicación; así también, se evaluó el control de malezas a los 10, 20, 30 y 40 días después de haber realizado la aplicación del herbicida en cada tratamiento según el procedimiento de Sánchez et al. (2015), y se calificó mediante la escala convencional de la Asociación Latinoamericana de Malezas (ALAM) (García y Mejía 2005). Se evaluaron un total de 12 caracteres agronómicos.

Mediante las combinaciones de los factores ensayados, se establecieron los siguientes tratamientos: 
Tabla 1. Tratamientos

\begin{tabular}{cccccc} 
Accent gr/ha & $\begin{array}{c}\text { Época de } \\
\text { Aplicación }\end{array}$ & $\mathrm{N}$ & \multicolumn{2}{c}{$\mathrm{P}_{2} \mathrm{O}_{5}$} & $\mathrm{~K}_{2} \mathrm{O}$ \\
\hline & $\mathrm{dds}$ & & & $\mathrm{Kg} / \mathrm{ha}$ & \\
$\mathrm{A}$ & 40 & 12 & 76 & 15 & 90 \\
$\mathrm{~B}$ & 40 & 12 & 152 & 30 & 180 \\
$\mathrm{C}$ & 40 & 18 & 76 & 15 & 90 \\
$\mathrm{D}$ & 40 & 18 & 152 & 30 & 180 \\
$\mathrm{E}$ & 40 & 24 & 76 & 15 & 90 \\
$\mathrm{~F}$ & 40 & 24 & 152 & 30 & 180 \\
$\mathrm{G}$ & 50 & 12 & 76 & 15 & 90 \\
$\mathrm{H}$ & 50 & 12 & 152 & 30 & 180 \\
$\mathrm{I}$ & 50 & 18 & 76 & 15 & 90 \\
$\mathrm{~J}$ & 50 & 18 & 152 & 30 & 180 \\
$\mathrm{~K}$ & 50 & 24 & 76 & 15 & 90 \\
$\mathrm{~L}$ & 50 & 24 & 152 & 30 & 180 \\
$\mathrm{M}$ & 60 & 12 & 76 & 15 & 90 \\
$\mathrm{~N}$ & 60 & 12 & 152 & 30 & 180 \\
$\mathrm{O}$ & 60 & 18 & 76 & 15 & 90 \\
$\mathrm{P}$ & 60 & 18 & 152 & 30 & 180 \\
$\mathrm{Q}$ & 60 & 24 & 76 & 15 & 90 \\
$\mathrm{R}$ & 60 & 24 & 152 & 30 & 180 \\
\hline
\end{tabular}

dds: días después de la siembra

\section{Resultados}

\section{Selectividad}

En las evaluaciones realizadas a los 3, 7, 14 y 21 días después de la aplicación del herbicida nicosulfuron en el cultivo de maíz, no se observó fitotoxicidad en las diferentes dosis; sino, únicamente en la evaluación realizada a los 3 días después de la aplicación, lo que se corrobora con lo descrito por (Gordillo et al., 2015).

En el aTabla 2, se presentan los índices de toxicidad a los 7 días después de la aplicación del Nicosulfuron; el análisis de variancia reportó alta significancia sólo para las dosis y épocas de aplicación del herbicida ensayado. El coeficiente de variación fue $22.5 \%$.

Los niveles de fertilización química se comportaron iguales estadísticamente, con valores de 3.16 y 3.0 para 152 - 30 - 180 y 76 - 15 $90 \mathrm{~kg} / \mathrm{ha} \mathrm{NPK}$, con calificaciones de daño moderado y poco daño, respectivamente. Las dosis de $40 \mathrm{~g} /$ ha Accent aplicado a los 12 días después de la siembra (dds.) y $40 \mathrm{~g}$ / ha Accent aplicado a los 18 dds. presentaron las calificaciones de 1.0; 1.0 y 2.0 respectivamente, es decir, poco daño, siendo iguales estadísticamente; difiriendo con las restantes dosis. Mientras que, las dosis 50 y 60 gr/ha Accent aplicado a los 24 dds. y 60 g/ ha Accent aplicado 
a los 18 dds. registraron un promedio de 5.0 equivalente a daño moderado, sin diferir significativamente.

Tabla 2.- Valores promedios del índice de toxicidad a los 7 días después de la aplicación en evaluación de diferentes dosis y épocas de aplicación del herbicida Accent (Nicolsulfuron)

en el cultivo de maíz (Zea mays) en presencia de dos niveles de fertilización química.

Babahoyo. Los Ríos. 2011.

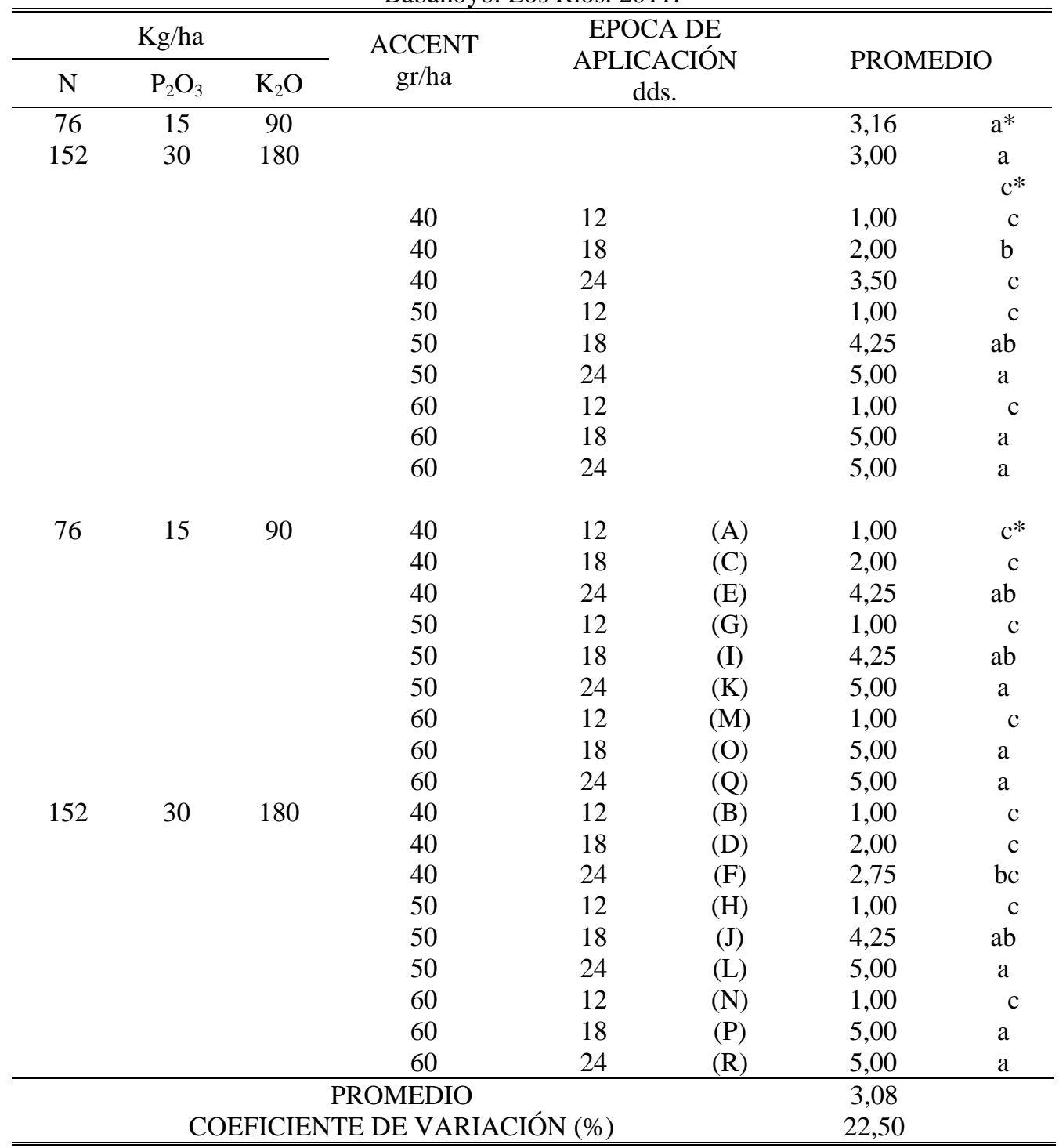

Promedios con una misma letra para niveles de fertilización no difieren

significativamente según prueba DMS; y para dosis y épocas de aplicación de herbicida e interacciones según prueba de Tukey al 95\% de probabilidad.

En el Tabla 3, se observan los promedios del índice de toxicidad en la evaluación realizada a los 14 días después de la aplicación. El análisis de variancia detectó alta significancia estadística para dosis y épocas de 
aplicación de Accent e interacciones; cuyo coeficiente de variación fue $16,99 \%$.

La prueba DMS, determinó igualdad estadística para los niveles de fertilización química, con una calificación de poco daño. La dosis de 40 g/ ha Accent aplicado a los 12 dds. fue inferior y diferente estadísticamente a las restantes dosis y épocas de aplicación; seguido de la dosis 50 y 60 g/ ha Accent aplicado a los 12 dds. con promedios de 1,12; 2.37 y 2,37 respectivamente; los dos últimos se comportaron iguales estadísticamente, difiriendo con las restantes dosis; con una calificación de poco daño.

La dosis de $40 \mathrm{~g} /$ ha Accent aplicado a los 12 dds. en presencia de los niveles 76 - 15 - 90 y 152 - 30 - $180 \mathrm{~kg} /$ ha NPK, presentó menor índice de toxicidad, es decir, más selectivo, con valor de 1,0 y 1,25 respectivamente, es decir produjeron poco daño a las plantas de maíz, lo cual concuerda con lo manifestado por (Delgado, Ortiz, y Zambrano 2006).

Cuadro 3.- Valores promedios del índice de toxicidad a los 14 días después de la aplicación en evaluación de diferentes dosis y épocas de aplicación del herbicida Accent

(Nicolsulfuron) en el cultivo de maíz (Zea mays) en presencia de dos niveles de fertilización química. Babahoyo. Los Ríos. 2011.

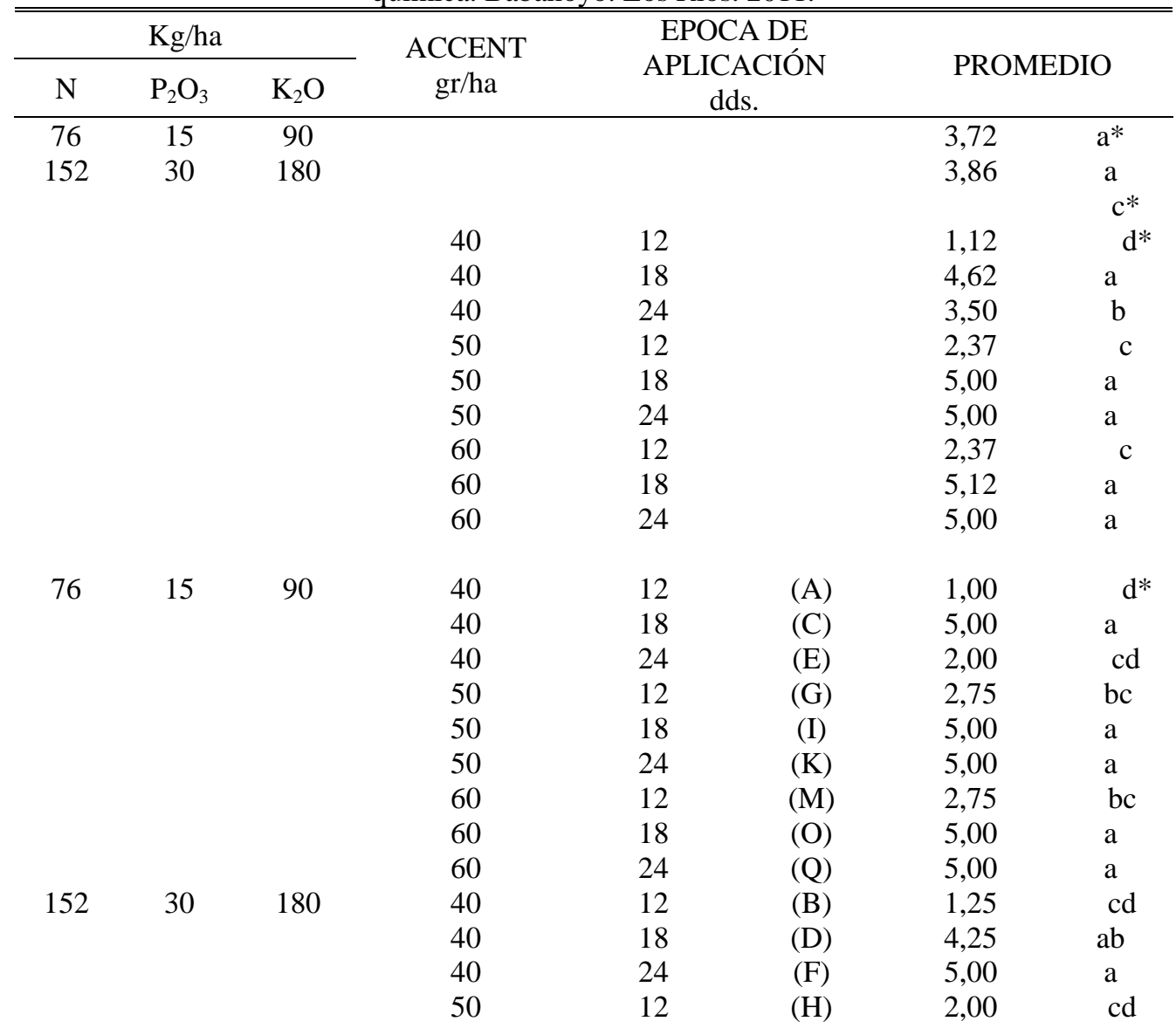




\begin{tabular}{ccccc}
50 & 18 & $(\mathrm{~J})$ & 5,00 & $\mathrm{a}$ \\
50 & 24 & $(\mathrm{~L})$ & 5,00 & $\mathrm{a}$ \\
60 & 12 & $(\mathrm{~N})$ & 2,00 & $\mathrm{~cd}$ \\
60 & 18 & $(\mathrm{P})$ & 5,25 & $\mathrm{a}$ \\
60 & 24 & $(\mathrm{R})$ & 5,00 & $\mathrm{a}$ \\
\hline PROMEDIO & & 3,79 & \\
\hline COEFICIENTE DE VARIACIÓN (\%) & & 16,66 & \\
\hline \hline
\end{tabular}

* $\quad$ Promedios con una misma letra para niveles de fertilización no difieren significativamente según prueba DMS; y para dosis y épocas de aplicación de herbicida e interacciones según prueba de Tukey al 95\% de probabilidad.

\section{Control de Malezas}

En el Cuadro 4, se presentan los promedios porcentuales de control de malezas en la evaluación realizada a los 10 días después de la aplicación del herbicida. El análisis de variancia detectó alta significancia estadística sólo para las dosis y épocas de aplicación del herbicida Accent; siendo el coeficiente de variación 5,97\%.

Entre los niveles de fertilización química no existió diferencia estadística, con promedios 69,86\% y 69,58\%; siendo un control bueno. La prueba de Tukey determinó igualdad estadística entre las dosis y épocas de aplicación del herbicida, a excepción de la dosis 40 g/ ha Accent aplicado a los 18 dds. que presentó el menor control con un valor de 63,12\%; los restantes fluctuaron de $70 \%$ a $71,87 \%$ resultando un buen control de malezas, concordando con lo expuesto por (Costar et al., 2013). Las interacciones entre niveles de fertilización química y épocas de aplicación del herbicida, no difirieron estadísticamente entre sí, a excepción de la dosis $40 \mathrm{~g} /$ ha Accent en presencia de $152-30-180 \mathrm{~kg} / \mathrm{ha}$ NPK, que registró el menor control de malezas en un 60\%. Cabe indicar que todas las interacciones, presentaron un buen control de malezas.

Cuadro 4.- Promedios porcentuales de control de malezas a los 10 días después de la aplicación en la evaluación de diferentes dosis y épocas de aplicación del herbicida Accent (Nicolsulfuron) en el cultivo de maíz (Zea mays) en presencia de dos niveles de fertilización química. Babahoyo. Los Ríos. 2011.

\begin{tabular}{ccccccc}
\hline \hline & Kg/ha & & $\begin{array}{c}\text { ACCENT } \\
\text { gr/ha }\end{array}$ & $\begin{array}{c}\text { EPOCA DE } \\
\text { APLICACIÓN } \\
\text { dds. }\end{array}$ & \multicolumn{2}{c}{ PROMEDIO } \\
\hline $\mathrm{N}$ & $\mathrm{P}_{2} \mathrm{O}_{3}$ & $\mathrm{~K}_{2} \mathrm{O}$ & & & & \\
\hline 76 & 15 & 90 & & & 69,86 & $\mathrm{a}^{*}$ \\
152 & 30 & 180 & & & 79,58 & $\mathrm{a}$ \\
& & & 40 & 12 & 71,87 & $\mathrm{a}^{*}$ \\
& & 40 & 18 & 63,12 & $\mathrm{~b}$ \\
& & 40 & 24 & 70,00 & $\mathrm{a}$ \\
& & 50 & 12 & 70,62 & $\mathrm{a}$ \\
& & 50 & 18 & 70,62 & $\mathrm{a}$ \\
& & 50 & 24 & $\mathrm{a}$
\end{tabular}




\begin{tabular}{|c|c|c|c|c|c|c|c|}
\hline & & & 60 & 12 & & 71,25 & $\mathrm{a}$ \\
\hline & & & 60 & 18 & & 70,00 & $\mathrm{a}$ \\
\hline & & & 60 & 24 & & 70,00 & a \\
\hline \multirow[t]{9}{*}{76} & 15 & 90 & 40 & 12 & (A) & 71,25 & $a^{*}$ \\
\hline & & & 40 & 18 & (C) & 66,25 & $a b$ \\
\hline & & & 40 & 24 & (E) & 70,00 & $a b$ \\
\hline & & & 50 & 12 & (G) & 70,00 & $a b$ \\
\hline & & & 50 & 18 & (I) & 70,00 & $a b$ \\
\hline & & & 50 & 24 & (K) & 70,00 & $a b$ \\
\hline & & & 60 & 12 & (M) & 71,25 & $\mathrm{a}$ \\
\hline & & & 60 & 18 & (O) & 70,00 & $a b$ \\
\hline & & & 60 & 24 & (Q) & 70,00 & $a b$ \\
\hline \multirow[t]{11}{*}{152} & 30 & 180 & 40 & 12 & (B) & 72,50 & $\mathrm{a}$ \\
\hline & & & 40 & 18 & (D) & 60,00 & b \\
\hline & & & 40 & 24 & (F) & 70,00 & $a b$ \\
\hline & & & 50 & 12 & (H) & 71,25 & $\mathrm{a}$ \\
\hline & & & 50 & 18 & $(\mathrm{~J})$ & 71,25 & $\mathrm{a}$ \\
\hline & & & 50 & 24 & (L) & 70,00 & $a b$ \\
\hline & & & 60 & 12 & $(\mathrm{~N})$ & 71,25 & $\mathrm{a}$ \\
\hline & & & 60 & 18 & (P) & 70,00 & $a b$ \\
\hline & & & 60 & 24 & (R) & 70,00 & $\mathrm{ab}$ \\
\hline & \multicolumn{4}{|c|}{ PROMEDIO } & \multicolumn{3}{|c|}{69,58} \\
\hline & \multicolumn{4}{|c|}{ COEFICIENTE DE VARIACIÓN (\%) } & \multicolumn{3}{|c|}{5,97} \\
\hline
\end{tabular}

Promedios con una misma letra para niveles de fertilización no difieren

significativamente según prueba DMS; y para dosis y épocas de aplicación de herbicida e interacciones según prueba de Tukey al 95\% de probabilidad.

\section{Conclusion}

El herbicida nicosulfuron en dosis de 40 g/ ha, mostró mayor selectividad al maíz.

El mejor control de malezas se logra a los 10 y 20 días después de la aplicación del nicosulfuron (Accent), en las dosis ensayadas.

Los niveles de fertilización química no influyeron en el índice de fitotoxicidad y control de malezas.

Las dosis y épocas de aplicación del herbicida nicosulfuron no influyeron significativamente en los caracteres agronómicos evaluados, a excepción de la variable índice de área foliar.

El índice de área foliar fue mayor con la dosis $50 \mathrm{~g} /$ ha Accent aplicado a los 12 días después de la siembra.

\section{References:}

1. Coello, R., y Cadena D. 2014. «Interacción entre herbicidas y distanciamientos de siembra en el cultivo de maíz, en la zona de Babahoyo». Tesis de Ingeniero Agrónomo, Babahoyo: Universidad Técnica de Babahoyo. 
2. Costar, A., J. Peña-Asín, M. Puig, A. Pérez, y A. Álvarez. 2013. «Relaciones de competencia entre maíz cultivado y las malas hierbas». Nutrición y Sanidad Vegetal.

3. Delgado, M., A. Ortiz, y C. Zambrano. 2006. «Resistencia de Rottboellia cochinchinensis (lour.) w.d. clayton al herbicida nicosulfuron en cultivos de maíz». Agrónomia Tropical 56 (2): 17182.

4. Fallas, Róger, F. Bertsch, C. Echandi, y C. Henríquez. 2010. «Caracterización del desarrollo y absorción de nutrimentos del híbrido de maíz HC-57». Agronomía Costarricense 35 (2). http://revistas.ucr.ac.cr/index.php/agrocost/article/view/6677.

5. Forti, R., y Gambino P. 1995. «Evaluación del momento de aplicación de nicosulfuron (4\% SC) para el control de malezas y la selectividad en el cultivo de maíz (Zea mays L.), utilizado sólo y en mezclas con cuatro herbicidas». Tesis de Master en Ciencias, Aragua, Venezuela: Universidad Central de Venezuela. Facultad de Agronomía.

6. García, Petra, y J. Mejía. 2005. «Control químico de malezas en maíz en un sistema de siembra directa». Agronomía Tropical 55 (3): 36380.

7. Gordillo, Fernando, C. Mejía, A. J. García, y C. Valencia. 2015. «Efecto de la dosificación de fertilizante sobre parámetros fotosintéticos de maíz medidos con la técnica fotoacústica». http://repositorio.uniquindio.edu.co/handle/123456789/526.

8. Hernández, M. M., J. M. Arreaza, y J. V. Lazo. 2002. «Evaluación de nicosulfuron en el control de Rottboellia exaltata, Euphorbia heterophylla y Aldama dentata en el cultivo de maíz (Zea mays L.) aplicado en tres diferentes estadios de desarrollo de las malezas». Revista de la Facultad de Agronomía 19 (4): 294-303.

9. Sánchez, Aymara, R. Delgado, J. Lorbes, V. Rodríguez, L. Figueredo, y C. Goméz. 2015. «Diagnóstico e índice para fertilización nitrogenada en etapa vegetativa del cultivo maíz (Zea mays L.)». Revista Unellez de Ciencia y Tecnología 33 (0). http://150.187.216.91/revistas/index.php/rucyt/article/view/320. 